\title{
Numerical Simulation of Sinusoidal Fluctuated Pulsatile Laminar Flow Through Stenotic Artery
}

\author{
A.B.M. Toufique Hasan ${ }^{1}$ and Dipak Kanti Das ${ }^{2}$ \\ ${ }^{I}$ Assistant Professor, ${ }^{2}$ Professor, Department of Mechanical Engineering, \\ Bangladesh University of Engineering and Technology(BUET), Dhaka-1000, Bangladesh. \\ E-mail:'toufiquehasan@me.buet.ac.bd,2'2ipak@me.buet.ac.bd
}

(Received July 18, 2007; accepted September 25, 2007)

\begin{abstract}
A numerical investigation is carried out for laminar sinusoidal pulsating flow through a modeled arterial stenosis with a trapezoidal profile up to peak Reynolds number of 1000. Finite element based numerical technique is used to solve the fluid flow governing equations where the fluid is assumed to be viscous, incompressible and Newtonian. The effects of pulsation, stenosis severity, Reynolds number and Womersley number on the flow behavior are studied. The dynamic nature of pulsating flow disturbs the radial velocity distribution and thus generates recirculation zone in the poststenotic region. The peak wall shear stress develops for 65\% stenosis (by area) is 3, 2.2, and 1.3 times higher than that for $30 \%, 45 \%$, and $55 \%$ stenosis, respectively. Peak wall shear stress and wall vorticity appear to intense at the throat of the stenosis. It is also observed that the peak wall vorticity seems to increase with the increase of stenosis size and Reynolds number. However, the peak values of instantaneous wall vorticity are not greatly affected by the variation of Womersley number.
\end{abstract}

Keywords: Stenosis, vorticity, wall shear stress, bio-fluid dynamics

\section{INTRODUCTION}

Investigation of the flow in a stenosed (constricted) geometry is of interest because of its significance in biomechanics especially in human vascular diseases. When too much cholesterol or high level of triglycerides (fatty substances) or both of them present in the blood, these fatty substances may begin to build as fatty streaks along the inside lining of the artery wall and causes the narrowing of the flow channel. Initially this streak grows very slowly. With course of time, as the hardening process and narrowing continues, the blood flow is disturbed. This thickening of stenotic artery was understood as an early process in the beginning of atherosclerosis which is the leading cause of human death in many countries. The haemodynamic behavior of the blood flow in arterial stenoses bears some important aspects due to engineering interest as well as feasible medical applications. In order to understand the effect of stenosis on blood flow through and beyond the constricted region of the artery, this has been the subject of much research both experimentally and numerically.

Numerical simulation of steady flow through axially symmetric rigid stenoses, with blood as a Newtonian fluid, have been reported by several investigators, among which are Deshpande et al. (1976), Lee (1990), Siegel et al. (1994), Reese and Thompson (1998). An integral interacting boundary layer theory for steady laminar flow in a asymmetrical bidimensional channel at high Reynolds number was presented by Largee et al.(2007). They accounted the effect of asymmetry of the geometry in the ideal fluid pressure expression and observed that the effect is small but noticeable. Though these simulations were based on steady state situation, but actually blood flow through arteries is inherently unsteady due to the cyclic nature of the heart pump and Reynolds number can vary from 100 to 1000 for medium to large sized artery. Berger and Jou (2000) reviewed the modeling studies and experiments on steady and unsteady, two and three dimensional flows in arterial geometries most relevant in the context of atherosclerosis. The technique of large-eddy simulation (LES) has been applied to the study of pulsatile flow through a modeled arterial stenosis by Mittal et al. (2001). They used a simple stenosis model that consist of a one-sided $50 \%$ semicircular constriction in a planner channel. The inlet volume flux is varied sinusoidally in a manner similar to the laminar flow simulations of Tutty (1992). LES is used to compute the flow at a peak Reynolds number of 2000 and a Strouhal number of 0.024. Mahapatra et al. (2002) numerically solved unsteady Navier-Stokes equations by finite difference technique in staggered grid distribution for a flow through a channel with locally symmetric and asymmetric constrictions. For flow through symmetric constriction the centerline vertical velocity exhibited finite oscillation behind the constriction at high Reynolds 
number. Zendehbudi and Moayeri (1999) presented the numerical solutions for a physiological pulsatile flow as well as for an equivalent simple pulsatile flow, having the same stoke volume as the physiological flow. The analysis was restricted to laminar flow, Newtonian fluid and axisymmetric rigid stenosis. Liao et al. (2003) studied numerically the physiological turbulent flow fields in the neighborhoods stenosed arteries for Reynolds numbers from 1000 to 4000 . The dimensionless diameter constriction ratios were allowed to vary from 0.375 to 0.6 for bell-shaped constrictions. The Womersley numbers were varied from 6 to 50 . The solution procedure was based on the method of artificial compressibility with implicit LU-SGS and used a decoupled approach to solve the Reynolds-averaged Navier-Stokes equations and $\mathrm{k}-\omega$ turbulence model equations.

Beside these, taking the non-Newtonian properties of the blood into consideration, there have been numerous studies. Tu and Deville (1996) simulated the blood flow through stenoses by incorporating the Herschel-Bulkley, Bingham and power-law fluids in the generalized incompressible Newtonian model. The geometry corresponded to a rigid circular tube with a partial occlusion. Ishikawa et al. (1998) used the bi-viscosity model as a constitutive equation for blood, and the flow was assumed to be periodic, incompressible and axisymmetric. They investigated the effect of pulsation and the rheological properties of blood. Also various mathematical models have been developed by a number of researchers (Jung et al., 2004; Chan et al., 2005 etc.) to simulate non-Newtonian blood flow through stenotic arteries.

Although the blood is known to be non-Newtonian in general, in the present study it is assumed to be Newtonian. This is because it is considered large arteries with radii of the order $1.0 \mathrm{~mm}$, where the velocity and shear rate are high. The apparent viscosity is nearly a constant in arteries with relatively large diameters $(\sim 5$ $\mathrm{mm}$ ), and therefore the non-Newtonian effects can be neglected. Again the assumption of rigid boundary may not seriously affect the results since development of atherosclerosis in arteries causes a considerable reduction in the elastic properties of its wall.

The present study is concerned with laminar pulsatile flow in a tube with symmetric stenosis, which represents a simplified model of blood flow in stenosed arteries and to investigate the effects of pulsation, constriction ratio, Reynolds number and Womersley number on the flow property.

\section{MATHEMATICAL MODELING}

For axisymmetric flow of incompressible and Newtonian fluids with constant fluid properties, the continuity and Navier-Stokes equations in two-dimensional cylindrical co-ordinates $(\mathrm{r}, \mathrm{Z})$ can be written in the differential conservation form as follows:

Continuity equation:

$$
\frac{1}{\mathrm{r}} \frac{\partial\left(\mathrm{rv}_{\mathrm{r}}\right)}{\partial \mathrm{r}}+\frac{\partial \mathrm{v}_{\mathrm{z}}}{\partial \mathrm{z}}=0
$$

r-direction momentum equation:

$$
\begin{array}{r}
\frac{\partial v_{r}}{\partial t}+v_{r} \frac{\partial v_{r}}{\partial r}+v_{z} \frac{\partial v_{r}}{\partial z}=-\frac{1}{\rho} \frac{\partial p}{\partial r}+ \\
\frac{\mu}{\rho}\left[\frac{\partial}{\partial r}\left(\frac{1}{r} \frac{\partial\left(r v_{r}\right)}{\partial r}+\frac{\partial^{2} v_{r}}{\partial z^{2}}\right)\right]
\end{array}
$$

Z-direction momentum equation:

$$
\begin{array}{r}
\frac{\partial v_{z}}{\partial t}+v_{r} \frac{\partial v_{z}}{\partial r}+v_{z} \frac{\partial v_{z}}{\partial z}=-\frac{1}{\rho} \frac{\partial p}{\partial z}+ \\
\frac{\mu}{\rho}\left[\frac{1}{r} \frac{\partial}{\partial r}\left(r \frac{\partial v_{z}}{\partial r}+\frac{\partial^{2} v_{z}}{\partial z^{2}}\right)\right]
\end{array}
$$

Here $\mathrm{r}$ and $\mathrm{z}$ are the radial and axial co-ordinates, respectively with the z-axis located along the axis of symmetry of the tube. The total velocity is defined by $v_{r}$ and $\mathrm{v}_{\mathrm{z}}$, the radial and axial components, respectively. The pressure, density and dynamic viscosity are denoted by $p, \rho$ and $\mu$, respectively. Fig. 1 shows the physical solution domain of the present problem where $\delta$ is the constriction radius (at the narrowest part of the stenosis) and $\mathrm{R}$ is the unconstricted radius.

The boundary conditions used in the present computations are:

- At the inlet:

$$
\begin{aligned}
& \mathrm{v}_{\mathrm{z}}=\frac{1}{\mathrm{~A}}\left[\overline{\mathrm{Q}}+\mathrm{P} \sin \left(2 \pi \frac{\mathrm{t}}{\mathrm{T}}\right)\right] \\
& \mathrm{v}_{\mathrm{r}}=0 .
\end{aligned}
$$

- At the outlet: $\frac{\partial \mathrm{v}_{\mathrm{r}}}{\partial \mathrm{z}}=\frac{\partial \mathrm{v}_{\mathrm{Z}}}{\partial \mathrm{z}}=0$

- At the centerline of the tube, axial symmetry condition.

- At wall, no-slip flow: $\mathrm{v}_{\mathrm{r}}=\mathrm{v}_{\mathrm{z}}=0$

Where $\mathrm{A}$ is the area of the unconstricted tube, $\overline{\mathrm{Q}}$ is the mean flow rate, $\mathrm{P}$ is the pulsating amplitude, $\mathrm{t}$ is time and $\mathrm{T}$ is the time period of the pulsatile flow.

\section{NUMERICAL SIMULATION}

\subsection{Computational Procedure}

Present numerical procedure is based on the finite element method (FEM). Mixed finite-element formulation (Reddy and Gartling 1994) for pressure and velocity is used. The starting point for the development of this finite element model of equations (1-3) is their weak forms. Then the velocity and pressure will be approximated by their respective interpolation functions. The computational domain is subdivided into finite number of elements. The elements used are isoparametric triangular. Discretization is performed in such a way that the numbers of elements are higher near the stenosis and also on the post-stenotic region where higher gradients of the variable values are expected. Quadratic interpolation function for velocity and linear interpolation function for pressure are used. From the 
linearization of continuity and momentum equations, large sets of linear algebraic equations are obtained. These linear algebraic equations are solved by direct linear solver UMFPACK (Unsymmetric Multi-frontal package). For temporal approximation, implicit approach is used for the present pulsating problem. The time stepping is determined by error estimation of all dependent variables between two successive time steps. Limiting criteria for determining the time steps is set as $1 \mathrm{e}-6$ for all dependent variables.

\subsection{Grid Independency Test}

A number of numerical computations have been performed using different blood vessel lengths upstream and downstream of the stenosis in order to ensure independence of the results from the position of the inflow and outflow boundaries. It is found that 10R and 40R lengths upstream and downstream, respectively, are sufficient. Again, since numerical results are greatly depends on the mesh generation, a grid sensitivity test is performed to find out the optimum number of elements to discretize the computational domain. Extensive tests are carried out with different sizes of mesh as given in table 1. 14,355 elements with 67,923 degrees of freedom has been selected to simulate the results based on accuracy and computational time as shown in Fig. 2.

\subsection{Validity of the Present Numerical Computation}

As the first step of analysis of the results, the present numerical computations must be validated with the several experimental and analytical solutions with comparable unsteady laminar flows in constricted tube. The numerical results are compared with the experiment of Ojha et al. (1989). Velocity profiles in the post stenotic regions will be compared for $z=1,2.5$ and 4.3, where $z$ is the normalized distance from the center of the stenosis; that is $z^{\prime}=\mathrm{z} / \mathrm{D}$, and $\mathrm{z}$ is the axial distance away from the center of the stenosis. The geometry used in the validation is a $45 \%$ stenosis (by area) with a trapezoidal profile. The pulsatile flow has waveform of $4.3 \pm 2.6 \mathrm{ml}$ with a period of $345 \mathrm{~ms}$ as shown in Fig. 3. In the experiment of Ojha et al. (1989), the pulsatile wave form was generated by a $2.9 \mathrm{~Hz}$ piston pump which was superimposed on that of a steady flow gear pump. There was a phase shift of $128^{0}$ (or $0.123 \mathrm{~s}$ time-shift) in the volumetric flow rate compared to present computation shown in Fig. 3. But this has been adjusted to their time scale in this section to make the comparison easier. The fluid has density of $755 \mathrm{~kg} / \mathrm{m}^{3}$ and a viscosity of 0.00143 $\mathrm{N} / \mathrm{m}^{2} \mathrm{~s}$ as deodorized kerosene (Shell-sol 715). Since blood is assumed to be Newtonian, the flow behavior for kerosene or blood will be identical for the same Reynolds number. The Reynolds number is defined as $\operatorname{Re}=\rho V_{\text {mean }} \mathrm{D} / \mu$, where $V_{\text {mean }}$ is the time-averaged mean velocity, $\mathrm{D}$ is the blood vessel inlet diameter, and $\mu$ is the limiting high shear rate Newtonian viscosity. This particular input pulse allows a direct change to the Womersley number, Wo $=R(2 \pi \rho / \mu \mathrm{T})^{1 / 2}$, the ratio of transient inertial effects to viscous effects, without altering the Reynolds number. The inlet pulse is sinusoidal pulse with a mean Reynolds number of 575 , and the maximum and minimum Reynolds numbers are 930 and 230, respectively. The Womersley number for this pulse is 7.75 .
To analyze velocity variations of post-stenotic region, the input centerline variations versus time are compared in Fig. 4(a). The inlet boundary condition is assumed by fully developed axial velocity profile. From the figure it can be seen that the computed inlet centerline velocities are similar throughout the pulse, expect near the peak of the pulse to that experimentally obtained. This additional acceleration in the experimental input pulse causes slight discrepancies with the data. Again post stenotic velocity profiles are used to validate the numerical computations with experimental data as shown in Fig. 4(b). The differences between the predictions and experimental data are found to be great near the zenith of the pulse as has been pointed out. However, the agreement is generally well.

\section{RESULTS AND DISCUSSIONS}

In this section, sinusoidal fluctuated pulsatile laminar flow through modeled stenosed artery will be discussed in order to investigate the effect of stenosis size, Reynolds number, Womersley number on the flow field. To do so, velocity distribution, streamlines of the flow field, wall vorticity, wall shear stress (WSS) under different geometrical and physical configuration will be high lightened. The flow waveform specified at the present model inlet is similar to Ojha et al. (1989) except that the experiment has a time-shift of $0.123 \mathrm{~s}$ (or phase shift of about $128^{0}$ ) to that of present computation.

\subsection{Radial Velocity Distribution in the Flow Field}

The presence of a stenosis in an artery can greatly influence on the flow field as shown in Fig. 5 for $55 \%$ stenosis, $\mathrm{Re}=575$ and $\mathrm{Wo}=10$. The axial velocity profiles for different positions at each time steps are significantly different from a parabolic velocity profile (Poiseuillean) for the Womersley number greater than one. Figure 5(a) shows the velocity distribution just upstream of the stenosis. At the beginning of the pulsated flow when $t / T=0$, the centerline velocity is slightly higher than that for steady flow (2 times the mean velocity). When $\mathrm{t} / \mathrm{T}=0.25$ that is for peak flow rate, the centerline velocity is approximately 3 times of mean flow velocity. Again at this time the instantaneous velocity is always higher for all radial positions. At the deceleration stage of the flow, when $t / T=0.5$, the centerline velocity is 2.6 times the mean velocity of flow. But region from the wall up to 0.3 times the radial distance of the artery is found where velocity is always lower than the steady velocity distribution. Beyond this region velocity is always higher. At $t / T=0.75$, for all the radial locations velocity is lower than the steady velocity distribution due to the significant reduction in flow at this time step. Here the center line velocity is 1.5 time the mean velocity.

Figure 5(b) shows the velocity distribution at the throat of the stenosis for different time steps. In this region, the fluid accelerates and velocity reaches its maximum. The velocity profile becomes flattened and reflects a boundary layer that is thin in comparison with the artery width. At time $\mathrm{t} / \mathrm{T}=0$ and 0.25 , the velocity gradient exists in very small region from the wall surface and beyond this region velocity became approximately uniform. The maximum velocity observed for these two 
times are 2.75 and 4.25 times the mean velocity. At time $\mathrm{t} / \mathrm{T}=0.5$, the centerline velocity is 3.1 times the mean one, whereas for $\mathrm{t} / \mathrm{T}=0.75$, the velocity throughout the radius of the artery is lower compared to other time steps.

Beyond the stenosis region the flow slows down and there may be boundary layer separation as shown in from Fig. 5(c). Due to sudden rise in area, the velocity distribution for all the time levels shows a region where flow velocity becomes approximately zero. This region is 0.3 times the radius of the artery. Beyond this region, velocity develops for all the time values.

Unsteady pulsated flow creates the disturbances in velocity distribution over the stenoses region. Velocity profile becomes very much flatter in the throat of the artery and downstream of the stenoses recirculation zone can be developed.

\subsection{Streamlines, Wall Vorticity and WSS for Mean $\operatorname{Re}=\mathbf{5 7 5}, \mathrm{Wo}_{\mathrm{o}}=10$}

Since the flow is pulsatile, the time-dependent flow field is more relevant for biomedical application. The instantaneous streamlines of the sinusoidal fluctuated flow show considerable variation through out the cycle as shown in Fig. 6 for $45 \%$ stenosis size. From Fig. 6 (a), at $\mathrm{t} / \mathrm{T}=0$, just beginning of the pulsated flow, the streamlines run smoothly through the stenosis flow field with the creation of a vortex very close to the post stenotic region. The separation and reattachment points are located to the inflection point of the wall curve. At this instant, the Reynolds number is equal to the mean one. After this time, the flow accelerates to the peak flow rate, the attached vortex grow in size and became fatter as shown in Fig. 6 (b) for $t / T=0.25$. When $t / T=0.5$, the instantaneous Reynolds number equals the mean one during decelerated flow of the total flux, the vortex occupies more space in the distal area. When the flow rate reaches its zero net flux and relatively low velocities at $\mathrm{t} / \mathrm{T}=0.75$, the remnants of these detached vortices occupy most of the constriction flow area, not only distal to the throat, but also proximal to the throat. After that, when $t / T$ advances to 1 , the next cycle restarts and the flow field follows these repeated pattern.

Though the streamlines show the pictures of the flow field in more detail, the flow behavior can also be presented in more detail by wall vorticity distribution for different time steps as shown in Fig. 7(a). The wall vorticity is also varied during the cycle, being higher than the wall vorticity of the steady flows under the same mean Reynolds number in some parts of the cycle, and lower in other parts. For all the time steps, the wall vorticity is highest in the constriction area than any other positions throughout the channel. The peak wall vorticity is observed for $\mathrm{t} / \mathrm{T}=0.25$ when the flow rate is peak. At time $t / T=0$ and $t / T=0.5$, the wall vorticity is also higher than the steady flow though the instantaneous Reynolds number is equal to mean one for these time levels.

Being the determining haemodynamic parameter in initiating the growth of atheroma, wall shear stress distribution along the axial direction are studied at different times during the cycle as shown in Fig. 7(b) for stenosis size of $55 \%$. Maximum wall shear stress occurred in the stenosis throat, which is consistent with observations frequently reported on steady flows. The magnitude of wall shear stress is largest at $t / T=0.25$ when flow is maximum in the cycle. The difference in upstream and downstream WSS in the post stenotic region indicates the creation of a vortex. The peak WSS for $\mathrm{t} / \mathrm{T}=0.25$ is 2.25 and 3 times higher to that for $\mathrm{t} / \mathrm{T}=$ 0 and 0.5 , respectively. At $t / T=0.75$, when net flux is zero shows a moderate rise in WSS at the stenosis throat.

\subsection{Effects of the Reynolds Number}

It is well known that the Reynolds number play a significant role on the flow field in the constricted tube for steady flow. The effect of the Reynolds number on the unsteady flow field will be discussed in the section. A constricted tube with $45 \%$ stenosis, Wo $=10$ with mean $\operatorname{Re}=150,300$ and 575 will be discussed.

The instantaneous streamlines for sinusoidally flow at Re $=150$ and 300 are shown in Fig 8(a) and 8(b), respectively. Referring to the streamlines in Fig. 6, 8(a) and $8(b)$, at the point of $t / T=0$, for $R e=150$, the streamlines run smoothly through the constriction flow field with no significant creation of vortex. However, for $\mathrm{Re}=300$ and 575 , there is a creation of a vortex in the streamlines. The size of the initial vortex is bigger for Re $=575$ compared to $R e=300$. At peak flow rate when $\mathrm{t} / \mathrm{T}$ $=0.25$, the attached vortex grows longer for $\mathrm{Re}=300$, and becomes shorter for $\operatorname{Re}=150$. After the peak flux, as the flow decelerates, the attached vortex still grows in size. Again for this time step $(t / T=0.5)$, the vortex size is small for $\mathrm{Re}=150$ compared to $\mathrm{Re}=300$ or $\mathrm{Re}=575$. At the time $t / T=0.75$ when the flow rate reaches is zero net flux, the remnants of the detached vortices occupy the distal to the throat as well as the proximal to the throat. However, the shortest vortex is observed for $\mathrm{Re}=$ 150.

Since the magnitude of the peak wall vorticity near the stenosis at peak flow rate is higher than the ones at other time steps, only the comparison of instantaneous wall vorticity distributions at $\mathrm{t} / \mathrm{T}=0.25$ is presented in Fig. 9 for brevity. The magnitude of maximum wall vorticity increases monotonically with the increase of Reynolds number. One more thing is observable that the location of the peak wall vorticity tends to shift slightly upstream as the Reynolds number increases. The peak wall vorticity for $\mathrm{Re}=575$ is 3 times higher than that for $\mathrm{Re}=$ 300. Again for $\mathrm{Re}=300$ and 575 , the wall vorticity downstream the stenosis does not seem to regain its undisturbed upstream values as it does for $\mathrm{Re}=150$. This is due to the increase in size of the recirculation region for higher Reynolds number.

It can be concluded that the flow pattern can be greatly influenced by the variation of the Reynolds number. Higher Reynolds number can cause the peak wall vorticity to increase sharply.

\subsection{Effect of Stenosis Size}

It is obvious that the stenosis size has a great influence on the flow field in constricted tube. In this section, the variation of the flow with the variation of stenosis size will be discussed. The numerical results are compared 
for four different stenosis sizes of $30 \%, 45 \%, 55 \%$ and $65 \%$. The Reynolds number is being fixed to 575 while the Womersley number is 10 .

The instantaneous streamlines for sinusoidal fluctuated flow at stenosis size of $30 \%, 55 \%$ and $65 \%$ are shown in Fig. 10(a), 10(b) and 10(c), respectively. Referring to the streamlines in Fig. 6,10 (a), 10(b) and 10(c), at $\mathrm{t} / \mathrm{T}=0$, for stenosis size of $30 \%$, the streamlines run smoothly through the stenosis flow field. However, for $65 \%$ stenosis, there is a bigger vortex development compared to other stenosis. At the peak flow rate when $\mathrm{t} / \mathrm{T}=0.25$, the attached vortex grows longer for $65 \%$ stenosis and becomes shorter for $30 \%$ stenosis. As the flow decelerates, the attached vortex continues to grow in size. When the flow rate reaches zero net flux at $t / T=$ 0.75 , the remnants of these detached vortices occupy distal to throat area. The shortest vortex is observed for $30 \%$ stenosis size. Unsteady flow field is more disturbed as the stenosis size increases.

Again, for the convenience of analysis, peak wall vorticity at peak flow rate is presented in Fig. 11. The value of maximum wall vorticity increases monotonically with increasing stenosis size. The value of maximum wall vorticity for stenosis size of $65 \%$ is approximately $3.25,2.15$, and 1.4 times higher than the corresponding values of vorticity when stenosis sizes are $30 \%, 45 \%$, and $55 \%$, respectively.

Figure 12 shows the variation of peak WSS for different stenosis severity at the time of maximum flow. The flow with the $65 \%$ stenosis is dominated by the wall shear stress especially in the stenosis position. For 30\% stenosis, the peak WSS is 15 pa and the downstream WSS regain the value of upstream WSS very quickly due to small disturbances created by this stenosis which is not observable for higher stenosis size. The peak WSS for $65 \%$ stenosis is $3,2.2$, and 1.3 times higher than that developed for $30 \%, 45 \%$, and $55 \%$ stenosis, respectively.

From these, it can be concluded that the severe stenosis size leads to a more complex flow field. The peak wall vorticity and WSS tend to increase with increasing stenosis size.

\subsection{Effects of the Womersley Number}

To investigate the effects of Womersley number, the details of the flow fields for different Womersley numbers are examined without varying the Reynolds number and the size of the stenosis. The Womersley numbers 5, 7.75, 10 and 12.5 are taken into consideration, with keeping the Reynolds number fixed to 575 and stenosis size of $45 \%$.

Figure 13 shows the instantaneous wall vorticity distribution at peak upstream flow rate when $\mathrm{t} / \mathrm{T}=0.25$ for the Womersley numbers of $5,7.75,10$, and 12.5 . It can be seen that the peak values of instantaneous wall vorticity are not greatly influenced by the variation of Womersley number. However, the wall vorticity downstream the stenosis for $\mathrm{Wo}_{\mathrm{O}}=12.5$ recovers rapidly to the same level as that of the constriction upstream, while this is not a case for the Womersley numbers of 5 , 7.75, and 10. For low Womersley number the recover process of wall vorticity is very slow.
The Womersley number can physically be interpreted as the ratio of unsteady to viscous force. The dynamic nature of the flow greatly depends on the frequency of the flow. The Womersley number does not greatly affect the maximum and mean wall vorticity distributions.

\section{CONCLUSIONS}

In this paper, a numerical simulation is presented to study the laminar flow through a modeled arterial stenosis. A simple axisymmetric model with a trapezoidal profile is chosen. The stenosis size is varied from $30 \%$ to $65 \%$ (by area). The flow at the artery inlet is taken as sinusoidal pulsated, and the peak Reynolds number was 1000 . The effect of pulsation, stenosis size, Reynolds number and Womersley number are investigated by comparing the results of instantaneous streamlines, instantaneous radial velocity distribution, instantaneous wall vorticity, and wall shear stress as well.

From the numerical investigation of sinusoidal fluctuated flows, the following conclusions can be drawn:

i. The recirculation zone in the pulsating flow changes in size with time, due to the variation of instantaneous flow rate.

ii. The flow deceleration in the pulsating cycles tends to enlarge the recirculation zone.

iii. The recirculation zones always prefer to occur simultaneously both the proximal and the distal to the stenosis when the instant net flow rate approaches to zero.

iv. The flow disturbances become more severe for higher stenosis size. The peak wall vorticity for $65 \%$ stenosis is $3.25,2.15$, and 1.4 times higher than that for $30 \%, 45 \%$ and $55 \%$, respectively. Similar impacts on other parameters are also found.

v. The variation of the Reynolds number can greatly influence the flow pattern. The peak wall vorticity is higher for higher Reynolds numbers than the ones for lower Reynolds numbers.

vi. Compared to other parameters, Womersley number does not have a significant influence on the peak values of wall vorticity.

\section{REFERENCES}

Berger, S.A., and Jou, L.D.(2000). Flows in stenotic vessels. Annual Review in Fluid Mechanics 32, 347-382.

Chan, W.Y., Ding,Y., and Tu, J.Y. (2007). Modeling of non-Newtonian blood flow through a stenosed artery incorporating fluid-structure interaction. ANZIAM Journal 47 (EMAC2005), C507-C523.

Deshpande, M.D., Giddens, D.P., and Mabon, R.F. (1976). Steady laminar flow through modeled vascular stenoses. Journal of Biomechanics 9, 165174. 
Ishikawa, T., Guimaraes, L.F.R., Oshima, S., and Yamane, R. (1998). Effect of non-Newtonian property of blood on flow through a stenosed tube. Fluid Dynamics Research 22, 251-264.

Jung, H., Choi, J.W., and Park, C.G. (2004). Asymmetric flows of non-Newtonian fluids in symmetric stenosed artery. Korea-Australia Rheology Journal $16,101-108$.

Lagree, P.-Y., Van Hirtum, A., and Pelorson, X.(2007). Asymmetrical effects in a 2D stenosis. European Journal of Mechanics B/ Fluids 26, 83-92.

Lee, T.S.(1990). Numerical studies of fluid flow through tubes with double constrictions. International Journal for Numerical Methods in Fluids 11, 11131126.

Liao, W., Lee, T.S., and Low, H.T. (2003). Numerical study of physiological turbulent flows through stenosed arteries. International Journal of Medical Physics C 14, 635-659.

Mahapatra, T.R., Layek, G.C., and Maiti, M.K. (2002). Unsteady laminar separated flow through constricted channel. International Journal of Nonlinear Mechanics 37, 171-186.

Mittal, R., Simmons, S.P., and Udaykumar, H.S. (2001). Application of large-eddy simulation to the study of pulsated flow in a modeled arterial stenosis. Journal of Biomechanical Engineering Transactions ASME 123, 325-332.
Ojha, M., Cobbold, R.S.C., Johnston, K.W., and Hummel, R.L. (1989). Pulsatile flow through constricted tube: an experimental investigation using photochromic tracer method. Journal of Fluid Mechanics 203, 173-197.

Reddy, J.N. and Gartling, D.K. (1994). The Finite Element Method in Heat transfer and Fluid Dynamics. Florida, USA.: CRC Press.

Reese, J.M., and Thompson, D.S. (1998). Shear stress in arterial stenoses: a momentum integral model. Journal of Biomechanics 31, 1051-1057.

Siegel, J.M., Markou, C.P., Ku, D.N., and Hanson, S.R. (1994).A scaling law for wall shear rate through an arterial stenosis. Journal of Biomechanical Engineering Transactions ASME 116, 446-451.

Tutty, O.R. (1992). Pulsatile flow in a constricted channel. Journal of Biomechanics 114, 50-54.

Tu, C., and Deville, M. (1996). Pulsatile flow of nonNewtonian fluids through arterial stenoses. Journal of Biomechanics 29, 899-908.

Zenedehbudi, G.R., and Moayeri, M.S.(1999). Comparison of physiological and simple pulsatile flows through stenosed arteries. Journal of Biomechanics 32, 959-965.

Table 1 Grid independency test for mean $\mathrm{Re}=575, \mathrm{Wo}=10.2$

\begin{tabular}{|c|c|c|c|c|}
\hline \multirow{2}{*}{$\begin{array}{c}\text { Number of elements } \\
\text { (degrees of freedom) }\end{array}$} & \multicolumn{4}{|c|}{ Peak Wall vorticity } \\
\cline { 2 - 5 } & $30 \%$ stenosis & $45 \%$ stenosis & $55 \%$ stenosis & $65 \%$ stenosis \\
\hline $7697(37957)$ & 100.55 & 148.78 & 225.2 & 307.32 \\
\hline $8475(41458)$ & 106.0 & 151.8 & 230.1 & 314.1 \\
\hline $10440(50303)$ & 106.0 & 152.3 & 238.78 & 316.25 \\
\hline $11776(56315)$ & 106.0 & 152.4 & 239.55 & 318.68 \\
\hline $14355(67923)$ & 106.0 & 152.4 & 239.5 & 318.64 \\
\hline $19640(91718)$ & 106.0 & 152.4 & 239.5 & 318.64 \\
\hline
\end{tabular}
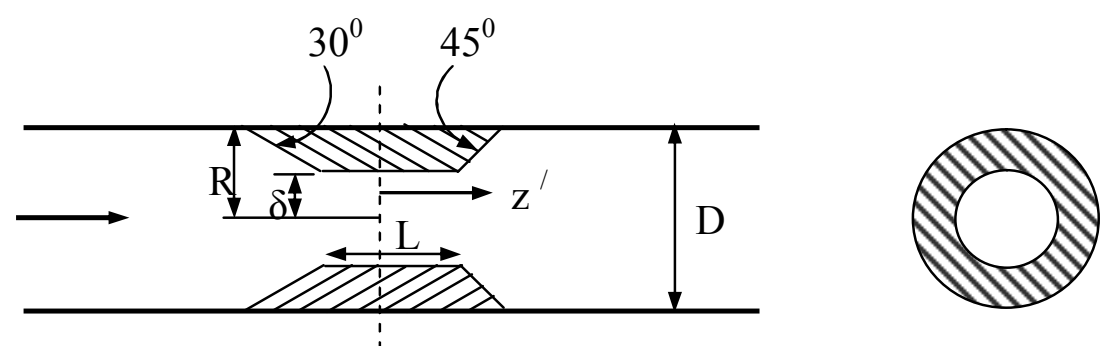

Fig. 1. Geometry of the stenosis used in the simulations, where $L=1.5 \mathrm{~mm}$ and $D=5.0 \mathrm{~mm}$. The normalized distance from the center of stenosis is given by $z=Z / D$. On the right is the cross-sectional view of the model at the throat. 


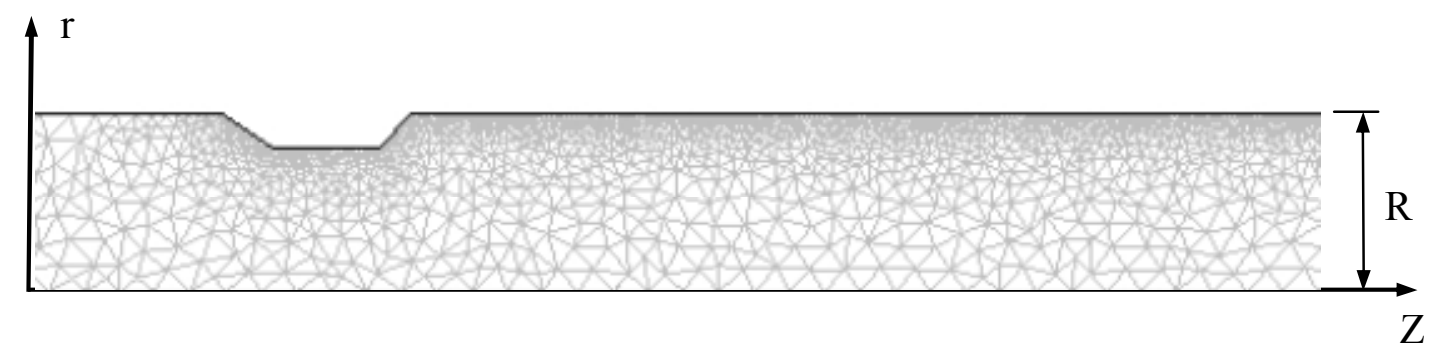

Fig. 2. Computational solution domain with finite element discritization of the present simulation

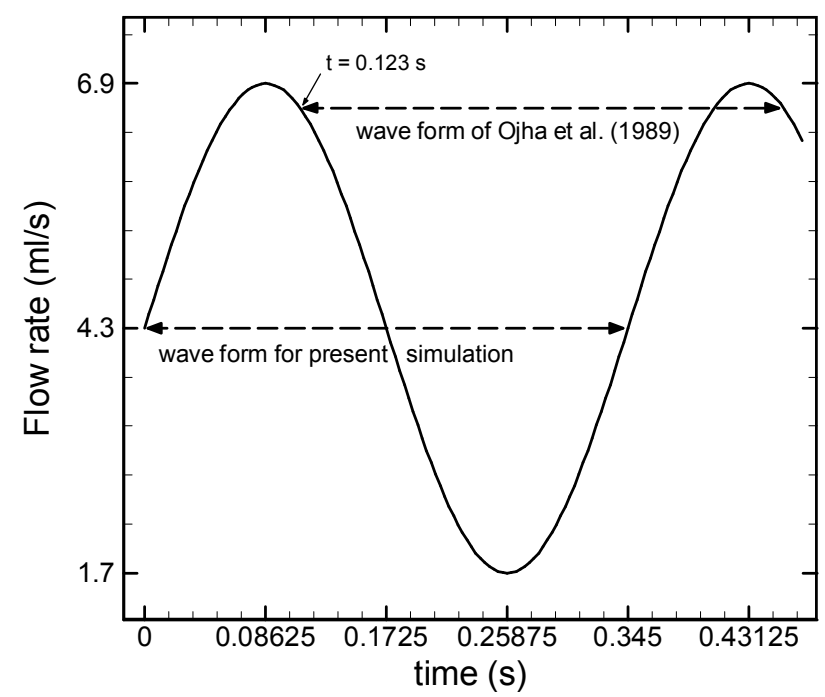

Fig. 3. The typical volumetric flow rate used in the simulation and that of Ojha et al. (1989)

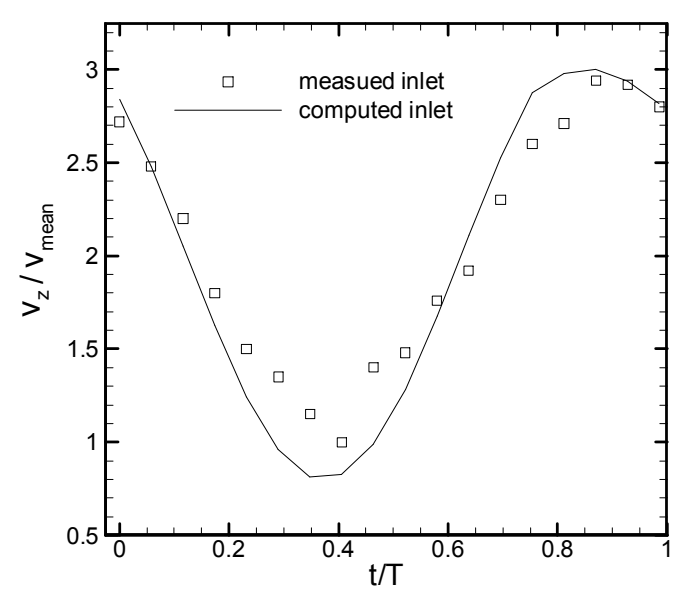

(a)

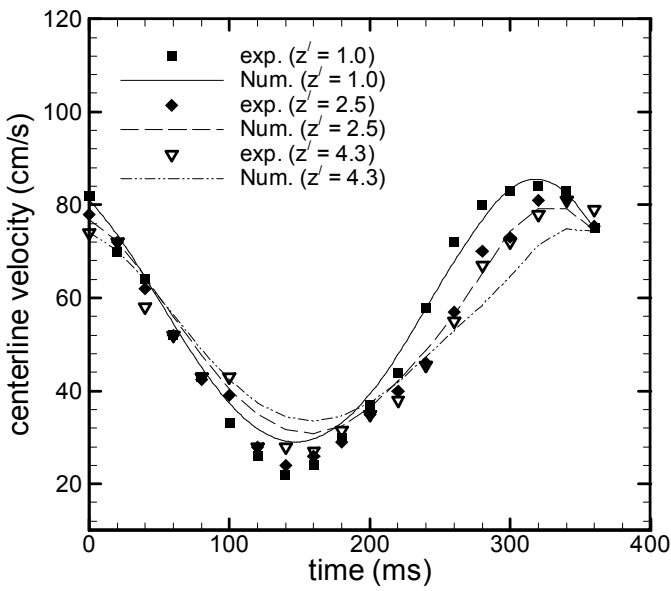

(b)

Fig. 4. Comparison of the present numerical computation with that carried out by Ojha et al.(1989), (a) inlet centerline velocity profile and (b) centerline axial velocity at different post stenotic region. 
A.B.M. Toufique Hasan and Dipak Kanti Das / JAFM, Vol. 1, No. 2, pp. 25 -35, 2008.

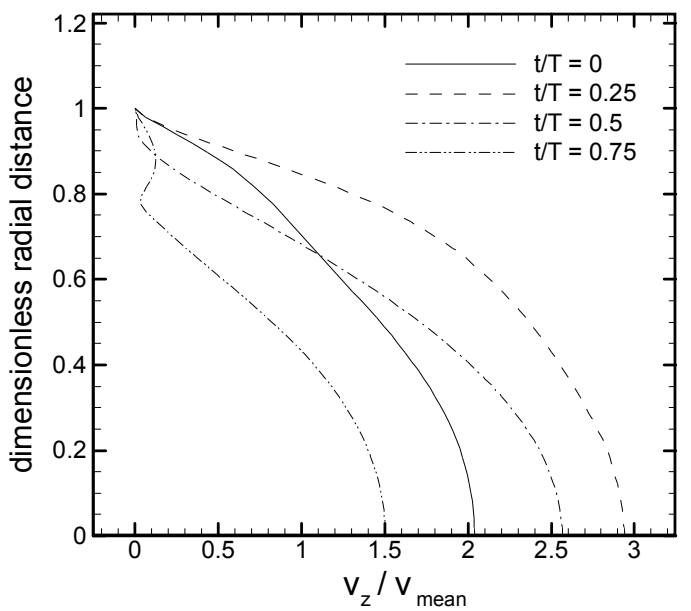

(a)

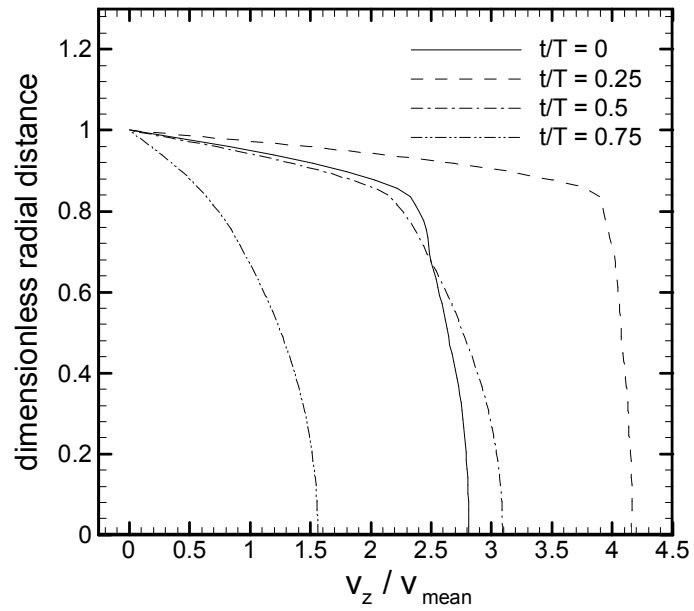

(b)

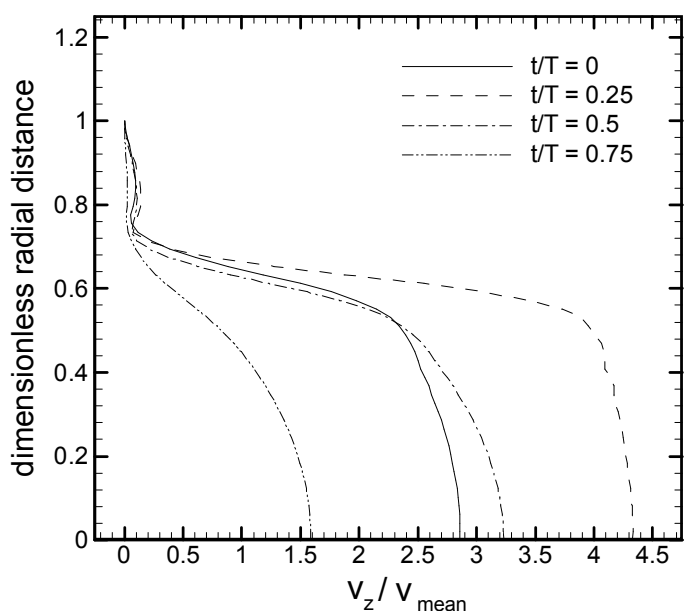

(c)

Fig. 5. Instantaneous radial velocity distribution for $55 \%$ stenosis size with mean $R e=575$, Wo $=10$, (a) just upstream of the stenoses, (b) throat of the stenoses and (c) just downstream of the stenoses.

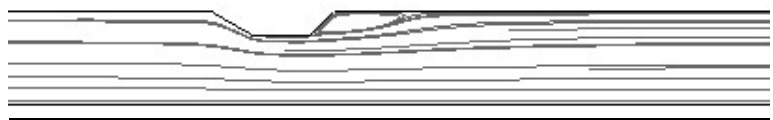

(a) $\mathrm{t} / \mathrm{T}=0$

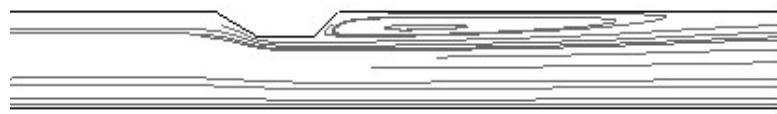

(b) $\mathrm{t} / \mathrm{T}=0.25$

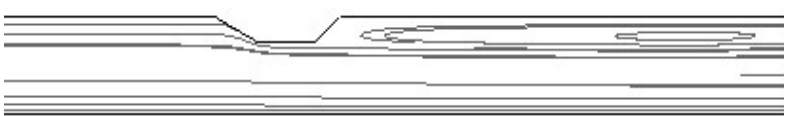

(c) $\mathrm{t} / \mathrm{T}=0.5$

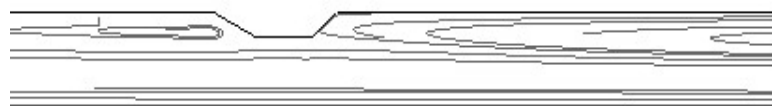

(d) $\mathrm{t} / \mathrm{T}=0.75$

Fig. 6. Instantaneous streamlines for sinusoidal fluctuated flow when mean $\mathrm{Re}=575$, $\mathrm{Wo}=10,45 \%$ stenosis. 
A.B.M. Toufique Hasan and Dipak Kanti Das / JAFM, Vol. 1, No. 2, pp. 25 -35, 2008.

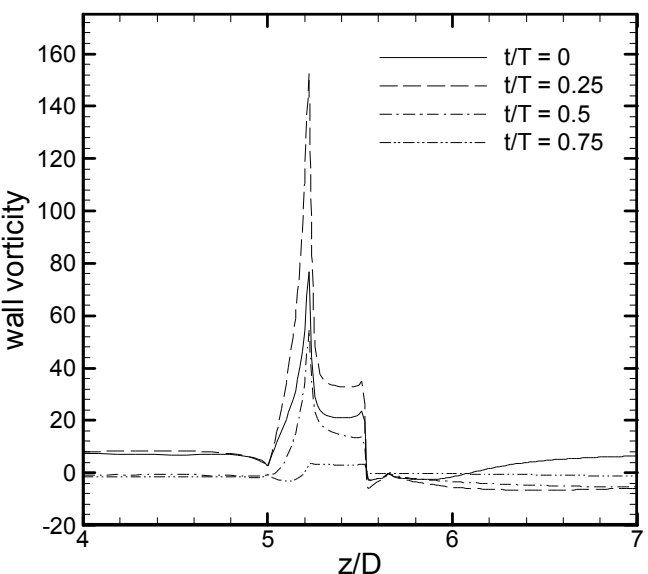

(a)

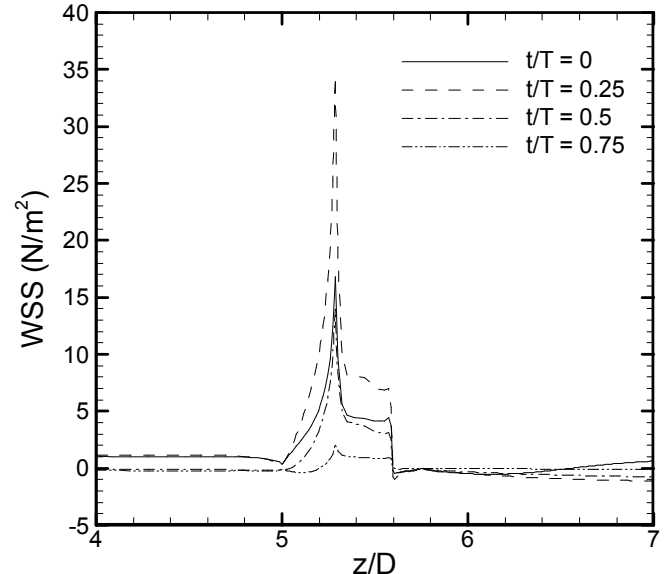

(b)

Fig. 7. Time dependent behavior of (a) wall vorticity of $45 \%$ stenosis and (b) wall shear stress of $55 \%$, for $\mathrm{Re}=$ $575, \mathrm{Wo}=10$.

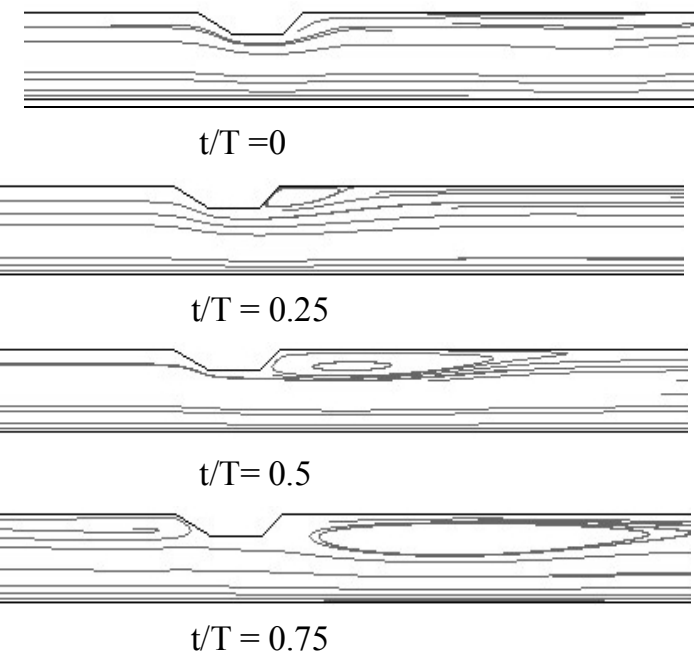

(a)

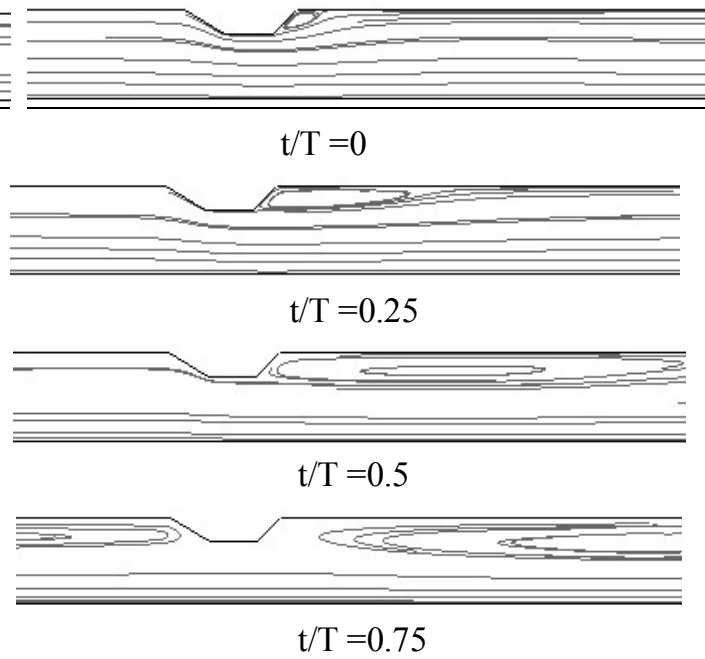

(b)

Fig. 8. Instantaneous streamlines for sinusoidal fluctuated flow when (a) $R e=150$ and (b) $300 ; 45 \%$ stenosis size, $\mathrm{Wo}=10$.

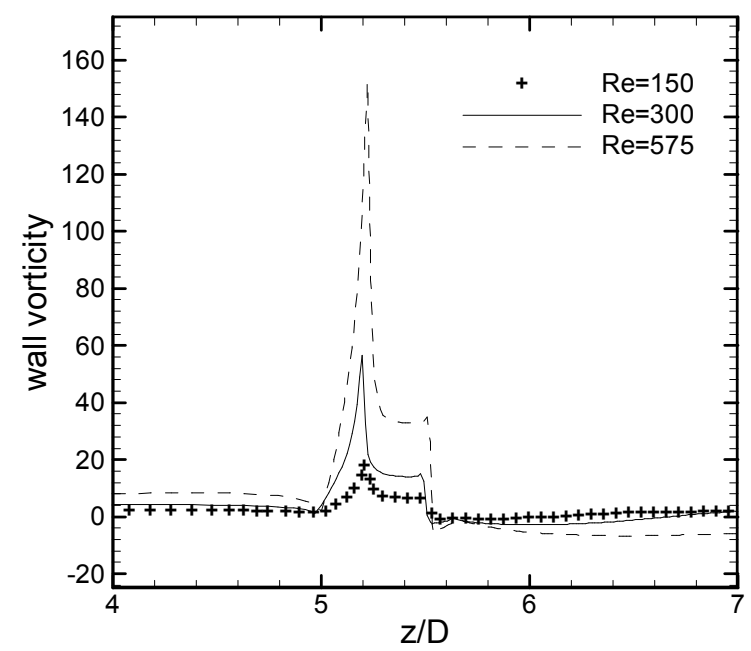

Fig. 9. Distribution of peak wall vorticity (at $\mathrm{t} / \mathrm{T}=0.25)$ of $45 \%$ stenosis $\left(\mathrm{Wo}_{\mathrm{o}}=10\right)$ at various axial locations for different Reynolds number 


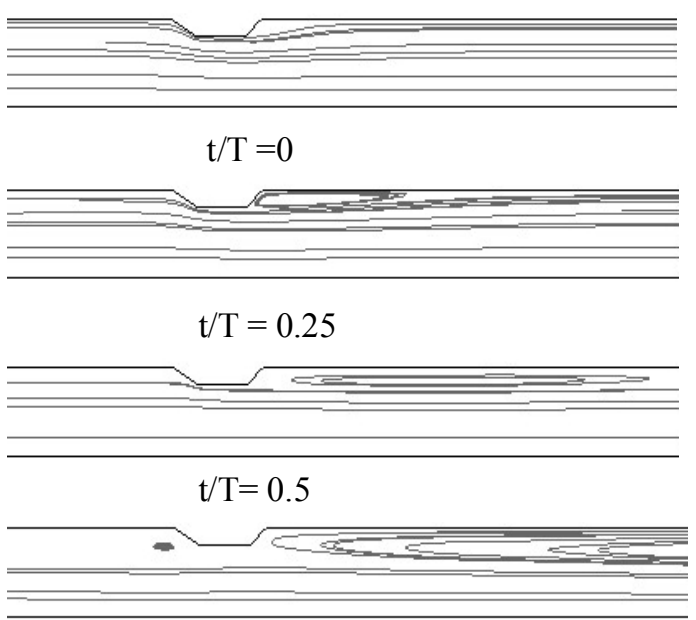

$\mathrm{t} / \mathrm{T}=0.75$

(a)

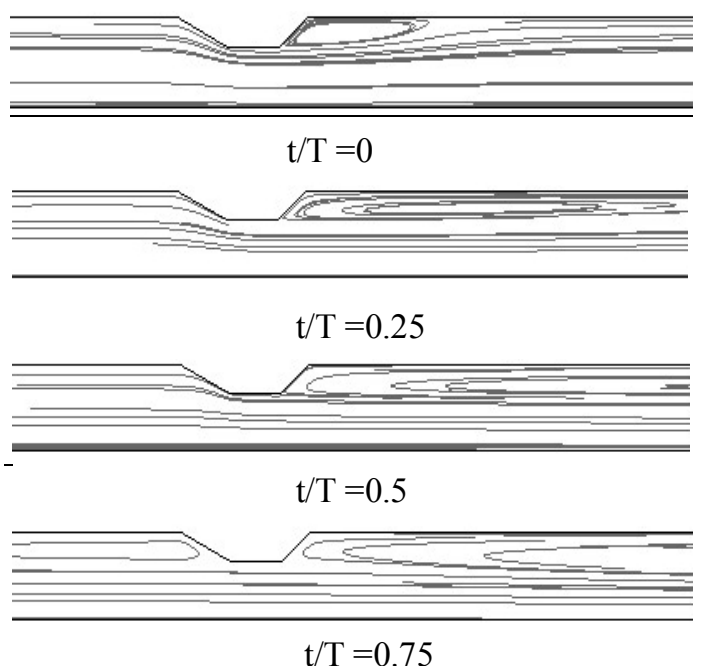

(b)

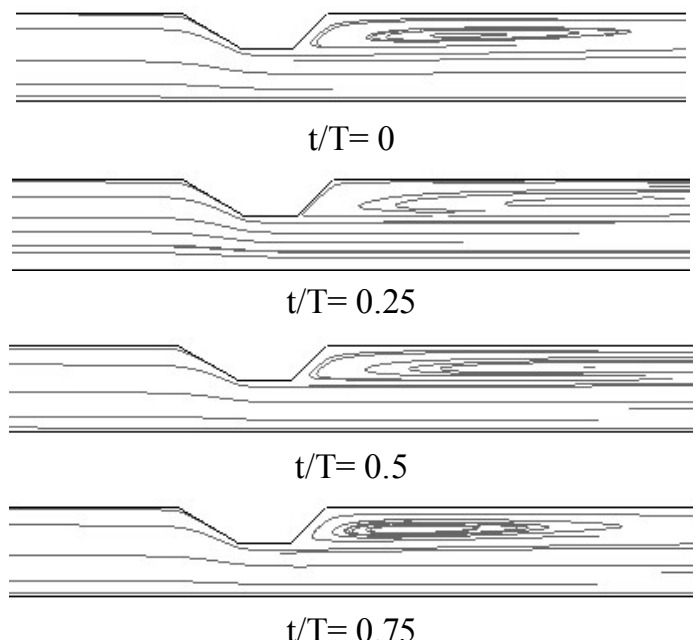

(c)

Fig. 10. Instantaneous streamlines for sinusoidal fluctuated flow when (a) $30 \%$ stenosis, (b) $55 \%$ stenosis, (c) $65 \%$ stenosis for mean $\mathrm{Re}=575, \mathrm{Wo}=10$.

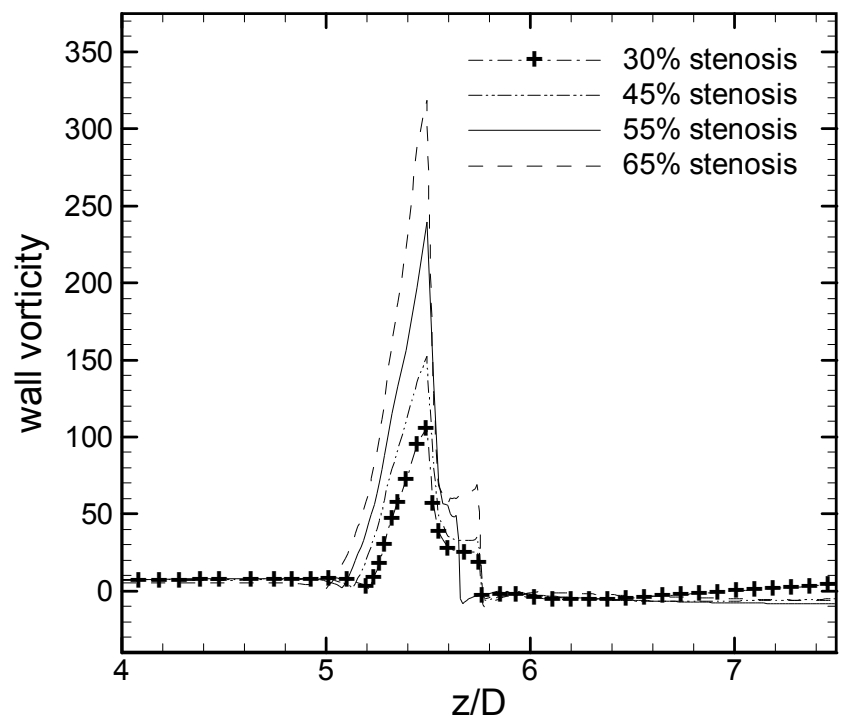

Fig. 11. Comparison of peak wall vorticity distribution for different stenosis sizes at mean $\mathrm{Re}=575, \mathrm{Wo}_{\mathrm{o}}=10$. 
A.B.M. Toufique Hasan and Dipak Kanti Das / JAFM , Vol. 1, No. 2, pp. 25 -35, 2008.

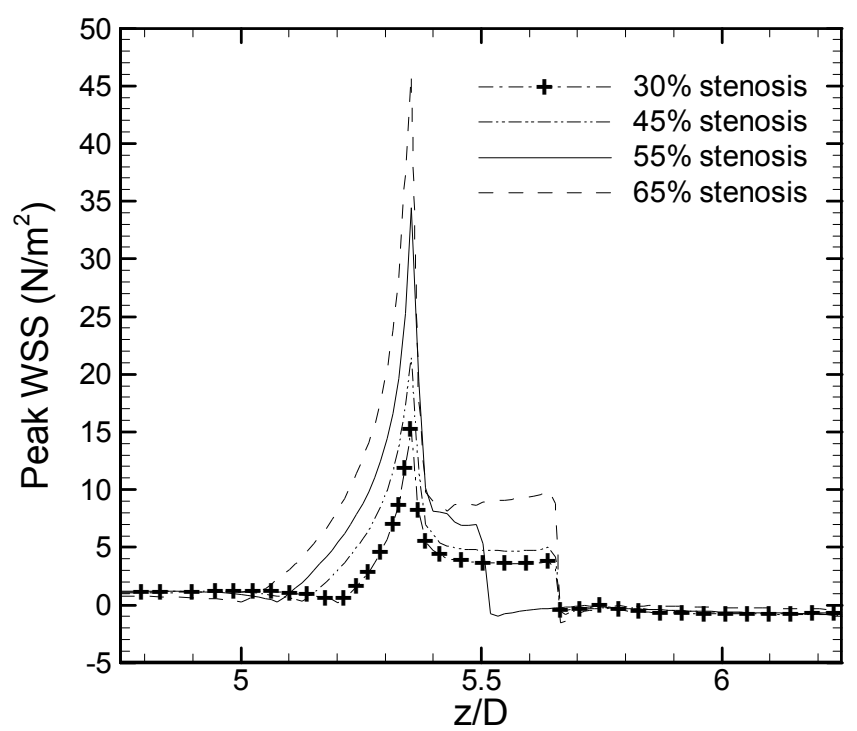

Fig. 12. Distribution of peak WSS for different stenosis size at mean $\mathrm{Re}=575, \mathrm{Wo}=10$.

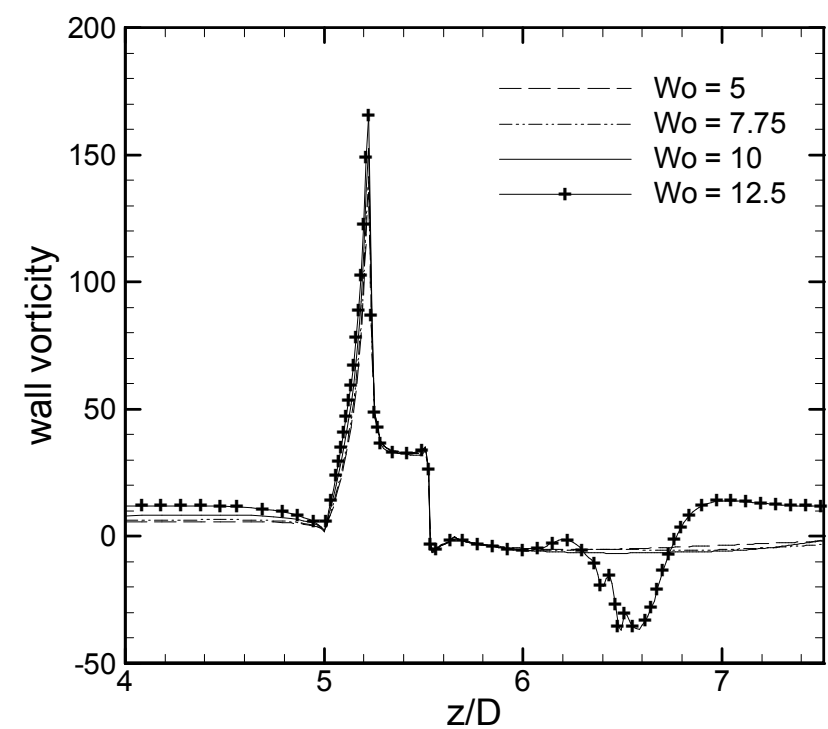

Fig. 13. Comparison of peak wall vorticity of $45 \%$ stenosis for different Womersley number at mean $\operatorname{Re}=575$ 